\title{
RESILIÊNCIA EM FAMÍLIAS DE PESSOAS COM ESQUIZOFRENIA: UM ESTUDO QUALITATIVO
}

\author{
Júlio Belo Fernandes \\ Doutor em Ciências de Enfermagem \\ Doutor em Nuevos Contextos de Intervención Psicológica en Educación, \\ Salud y Calidad de Vida \\ Escola Superior de Saúde Egas Moniz / Centro de Investigação Interdisciplinar Egas Moniz (CiiEM) \\ juliobelo@sapo.pt \\ Sónia Belo Fernandes \\ Doutora em Nuevos Contextos de Intervención Psicológica en Educación, \\ Salud y Calidad de Vida \\ Mestre em Saúde Pública \\ Projetar Enfermagem \\ Florencio Vicente Castro \\ Profesor Doctor Catedrático en la Facultad de Educación \\ Universidad de Extremadura
}

Recepción Artículo: 15 septiembre 2020

Admisión Evaluación: 22 octubre 2020

Informe Evaluador 1: 25 noviembre 2020

Informe Evaluador 2: 23 noviembre 2020

Aprobación Publicación: 27 noviembre 2020

\section{RESUMO}

Enquadramento: Ser familiar cuidador de uma pessoa com esquizofrenia é uma função complexa, que pode conduzir ao desenvolvimento de tensões no sistema familiar e, consequentemente, a dificuldades na adaptação ao papel de cuidador. É fundamental o desenvolvimento da resiliência dos familiares cuidadores perante esta adversidade, de modo a que estes sejam capazes de recuperar, resistir ou adaptar-se às exigências do cuidado. Objetivo: Identificar e explorar as barreiras à resiliência familiar em cuidadores de pessoas com esquizofrenia. Método: Foi realizado um estudo qualitativo com recurso a entrevistas semiestruturadas a 18 familiares cuidadores de pessoas com esquizofrenia que recebem apoio de uma Instituição Particular de Solidariedade Social da região de Lisboa e Vale do Tejo. Os dados foram analisados com recurso à técnica de análise de conteúdo. Resultados: A maioria dos participantes é do género feminino $(77,8 \%)$ e vive com 0 familiar de quem cuida (77,8\%). 0 papel de cuidador é assumido principalmente pela mãe (77,8\%). As barreiras à resiliência familiar enquadram-se amplamente em três categorias, nomeadamente dimensão emocional, dimensão relacional e dimensão racional, que se dividem em seis subcategorias: auto-estigmatização, emoção expressa, afastamento relacional, défice de conhecimentos, culpabilização e autoculpabilização. Conclusão: A identificação de barreiras à resiliência familiar fornece uma visão das dificuldades vividas pelos cuidadores, contribuindo para que os 


\section{RESILIÊNCIA EM FAMÍLIAS DE PESSOAS COM ESQUIZOFRENIA: UM ESTUDO QUALITATIVO}

profissionais de saúde se foquem nesses fatores e implementem estratégias que possibilitam remover ou minimizar a sua influência, potenciando as capacidades da pessoa para alcançar a adaptação positiva.

Palavras-chave: barreiras; resiliência psicológica; família; esquizofrenia; pesquisa qualitativa

\section{ABSTRACT}

Resilience in family of people with schizophrenia: A qualitative study-Background: Being a family caregiver of a person with schizophrenia is a complex responsibility, which can lead to the development of tensions in the family system and, consequently, difficulties in adapting to the role of caregiver. It is essential to develop family resilience in the face of this adversity, so that they are able to recover, resist or adapt to the demands of care. Aim: To identify and explore the barriers to family resilience in family caregivers of people with schizophrenia. Methods: A qualitative design, centred on the opinions of 18 family caregivers of patients with schizophrenia who receive support from a Private Institution of Social Solidarity in the region of Lisbon and Vale do Tejo. Data were analysed using the content analysis technique. Results: The majority of participants were female $(77.8 \%)$ and lived with and cared for their relative (77.8\%). The role of caregiver was assumed mainly by the mother $(77.8 \%)$. Barriers to family resilience broadly fall under three categories, emotional dimension, relational dimension and rational dimension, which are divided into six subcategories: self-stigmatization, expressed emotion, relational withdrawal, lack of knowledge, blame and self-blame. Conclusion: The identification of barriers to family resilience gives an insight into the difficulties experienced by caregivers, allowing health professionals to focus on these factors and implement a strategy that allows removing or minimizing their influence, enhancing the person's abilities to achieve positive adaptation.

Keywords: barriers; resilience; psychological; family; schizophrenia; qualitative research

\section{INTRODUÇÃO}

Na sociedade atual a função de cuidador informal da pessoa com esquizofrenia recaí sobre a família, que assume as responsabilidades do cuidado, tradicionalmente prestado por instituições de saúde. Esta mudança causa elevado impacto na dinâmica familiar, com repercussões a nível emocional e económico (Caqueo-Urízar, Rus-Calafell, Urzúa, Escudero, \& Gutiérrez-Maldonado, 2015).

Com 0 diagnóstico de esquizofrenia, os familiares cuidadores experienciam uma variedade de emoções negativas à medida que tentam lidar com a doença (McCann, Lubman, \& Clark, 2011). A função de familiar cuidador é geralmente difícil, pois o comportamento causado pela doença, bem como todas as conotações culturais e sociais negativas, conduzem ao desenvolvimento de tensões no sistema familiar e, consequentemente, a dificuldades na adaptação ao papel de cuidador (Caqueo-Urízar et al., 2015; Kuipers, Onwumere, \& Bebbington, 2010). No entanto, apesar de enfrentar muitas dificuldades, alguns familiares assumem o papel de cuidadores mais facilmente do que os outros, dependendo essa resposta de diversos fatores.

Tendo em conta esta problemática é fundamental potenciar o desenvolvimento da resiliência dos familiares cuidadores perante as adversidades decorrentes da doença.

As pesquisas nesta área estão concentradas cada vez mais nos cuidadores que se demonstram resilientes, na medida em que são capazes de recuperar, resistir ou adaptar às exigências do cuidado (Herrman et al., 2011)

0 estudo da resiliência familiar permite que o foco deixe de estar centrado no processo saúde/doença da pessoa, passando a existir uma abordagem que inclui a família e a comunidade, articulando as relações entre os contextos sociais, culturais, económicos e políticos (Eckermann, 2018).

A resiliência familiar é importante, porque é um processo que permite superar as situações adversas, promovendo um bem-estar familiar (Fernandes, \& Castro, 2019). Refere-se à capacidade de superar crises e retornar a um estado funcional a partir de um estado disfuncional, ou de recuperar funções diante de adversidades. A resiliência não pode ser encarada como uma característica específica de determinada pessoa ou uma construção estática, mas um processo dinâmico que muda com o tempo e 0 ambiente (Kim, Lim, Kim, \& Kim, 2018). Este 
processo pode ser desencadeado e desaparecer em determinados momentos da vida, bem como estar presente em algumas áreas e ausente noutras (Rutten et al., 2013).

Diversos autores desenvolveram teorias que exploram a construção da resiliência, variando o foco de acordo com a população examinada e a perspectiva do investigador. Apesar dessas diferenças, a maioria das teorias centram-se nos conceitos de adversidade e adaptação positiva (Teahan et al., 2018).

A forma como cada pessoa reage perante uma adversidade pode variar consideravelmente, esta pode ser aguda ou cumulativa, dependendo das experiências individuais e do curso de vida, pelo que os resultados negativos comuns relatados pelos cuidadores devem ser encarados como a adversidade a que estes se devem adaptar ou superar (O'Dwyer, Moyle, Taylor, Creese, \& Zimmer-Gembeck, 2017).

Um dos modelos explicativos do processo de resiliência foi desenvolvido por Patterson e Kelleher (2005). Este modelo consiste em 4 fases: deterioração, adaptação, recuperação e crescimento. Segundo estes autores, alguns cuidadores podem adaptar-se à adversidade, mas não conseguem recuperar completamente, outros cuidadores atingem a fase de recuperação e retornam ao seu nível de funcionamento, existindo ainda cuidadores que prosperam como consequência da superação da adversidade com que se depararam.

Dado que a adversidade deve ser superada, esta investigação adota o ponto de vista de que 0 cuidar por si só não é uma adversidade, mas sim os resultados negativos associados aos cuidados, os quais impedem os cuidadores de se adaptarem ou superarem positivamente, de modo a alcançar a fase de recuperação ou crescimento.

Diversos profissionais de saúde reconhecem que 0 estudo da resiliência familiar pode possibilitar 0 desenvolvimento de estratégias de prevenção e intervenção (Li, Qiao, Luan, Li, Wang, 2019). Existem diversos fatores que podem atuar como barreiras à resiliência, mesmo nas piores circunstâncias (Melillo, \& Suárez-0jeda, 2005). Essas barreiras podem ser diferentes em relação a uma variedade de contextos (Davydov, Stewart, Ritchie, \& Chaudieu, 2010). A influência desses fatores para um domínio da saúde não implica, por si só, um papel noutros. A sua especificidade em relação a domínios específicos ainda não foi esclarecida (Luthar, Cicchetti, \& Becker, 2000).

Há uma escassez de estudos que permitam identificar e compreender esses fatores. Esta pesquisa tem como objetivo identificar e explorar as barreiras à resiliência familiar em cuidadores de pessoas com esquizofrenia.

\section{MÉTODO}

A opção metodológica que orientou este estudo foi uma abordagem qualitativa, descritivo-exploratória. A população é constituída pelos familiares cuidadores de pessoas com esquizofrenia que recebem apoio de uma Instituição Particular de Solidariedade Social da região de Lisboa e Vale do Tejo.

0 método de amostragem selecionado foi a amostragem não probabilística por conveniência.

Os critérios de inclusão delineados foram: 0 diagnóstico de esquizofrenia ter sido realizado há cinco ou mais anos; ser cuidador informal da pessoa com esquizofrenia há cinco ou mais anos; saber ler e escrever em português.

Todos os familiares cuidadores de pessoas com esquizofrenia disponíveis no momento da colheita de dados e que cumpriam os critérios de inclusão foram incluídos na investigação com o intuito de permitir obter a maior variabilidade possível de dados.

\section{Participantes}

Após a recolha dos dados obteve-se uma amostra constituída por 18 familiares cuidadores de pessoas com esquizofrenia. Pela análise da tabela 1 constata-se que a maioria dos participantes são do género feminino (77,8\%), a média de idades foi 58,6 anos e o desvio padrão foi 10,182 anos. No que respeita ao grau de coabitação, a maioria dos participantes $(77,8 \%)$ vive com o familiar de quem cuida e o papel de cuidador é assumido principalmente pela mãe $(77,8 \%)$, sendo 0 pai 0 principal cuidador em apenas $11,1 \%$ das situações. 
Tabela 1. Características dos participantes

\begin{tabular}{|c|c|c|c|}
\hline & & $\begin{array}{c}\text { Frequênc } \\
\text { ia }\end{array}$ & $\begin{array}{c}\text { Percentage } \\
\text { m }\end{array}$ \\
\hline \multirow{2}{*}{ 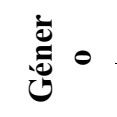 } & Feminino & 14 & 77,8 \\
\hline & Masculino & 4 & 22,2 \\
\hline \multirow{3}{*}{ 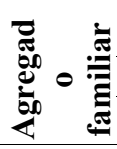 } & 2 & 6 & 33,3 \\
\hline & 3 & 8 & 44,5 \\
\hline & 4 & 4 & 22,2 \\
\hline \multirow{4}{*}{ 导 总 } & Mãe & 14 & 77,8 \\
\hline & Pai & 2 & 11,1 \\
\hline & Filho/filha & 1 & 5,55 \\
\hline & Irmão/irmã & 1 & 5,55 \\
\hline \multirow{6}{*}{ } & Média & \multicolumn{2}{|c|}{58,6} \\
\hline & Mediana & \multicolumn{2}{|c|}{61,5} \\
\hline & Moda & \multicolumn{2}{|r|}{62} \\
\hline & Desvio padrão & \multicolumn{2}{|c|}{10,182} \\
\hline & Mínimo & \multicolumn{2}{|r|}{30} \\
\hline & Máximo & \multicolumn{2}{|r|}{70} \\
\hline
\end{tabular}

\section{Recolha de dados}

Todos os participantes foram entrevistados pelo investigador principal numa sala privada da instituição. Foram realizadas entrevistas semiestruturadas com um tempo médio de 20 minutos.

Para a realização das entrevistas foi organizado um guião, desenvolvido com base nas evidências encontradas na revisão da literatura, tendo a contribuição de investigadores peritos em metodologia de investigação qualitativa. Previamente à sua aplicação, este foi devidamente validado. As questões pré-formuladas foram apresentadas aos participantes segundo a ordem que foi mais conveniente no decurso da entrevista, sendo o esquema de entrevista flexível e com função de auxiliar de memória.

As entrevistas foram gravadas em formato áudio digital e posteriormente transcritas na totalidade. Cada entrevistado teve acesso ao seu contributo de forma a corroborar o rigor da transcrição.

0 conjunto total de dados verbais recolhidos resultantes de cada entrevista foi armazenado em formato Microsoft Word®. De forma a garantir 0 anonimato e a confidencialidade dos entrevistados, foi atribuído um código numérico a cada entrevistado, seguido por números consecutivos de acordo com a ordem cronológica das entrevistas.

\section{Análise de dados}

0 processo de análise de dados foi realizado aplicando a análise conteúdo, conforme descrito por Bardin (2004), que envolveu pré-análise, codificação, categorização e interpretação dos dados. Esse processo foi orientado com o objetivo de identificar temas que permitissem responder à questão de pesquisa.

As gravações das entrevistas foram escutadas várias vezes para obter um senso geral. Após a transcrição em dados textuais, o texto foi dividido em unidades de significado, envolvendo palavras e frases sobre o mesmo tópico. Usando as próprias palavras dos participantes, os códigos foram atribuídos às unidades de significado. 
Categorias e subcategorias foram identificadas com base em diferenças e semelhanças. Os investigadores realizaram as etapas da análise separadamente, sendo que o consenso se alcançou através de discussão. A análise qualitativa não foi realizada com recurso a um programa informático porque a equipa de investigação preferiu a abordagem tradicional para essa análise.

\section{Considerações éticas}

Antes da realização deste estudo, um protocolo de pesquisa foi analisado e aprovado pelo Conselho Diretivo da instituição. Todos os procedimentos seguidos foram realizados em conformidade com a Declaração de Helsínquia de 1964 e as suas alterações posteriores ou princípios éticos comparáveis. Além disso, foi obtido 0 consentimento informado de todos os participantes para gravar a entrevista, relatar e publicar anonimamente os dados recolhidos. Após a transcrição literal, todos os dados registrados foram destruídos.

\section{RESULTADOS}

Após análise dos dados, foram identificadas diversas barreiras à resiliência familiar, as quais foram agrupadas em três categorias, que se dividem em seis subcategorias (Tabela 2).

Tabela 2. Categorias e subcategorias

\begin{tabular}{cc}
\hline Categoria & Subcategoria \\
\hline \multirow{2}{*}{ Dimensão Emocional } & Auto-estigmatização \\
\cline { 2 - 2 } & Emoção expressa \\
\hline Dimensão Relacional & Afastamento relacional \\
\hline \multirow{2}{*}{ Dimensão Racional } & Défice de conhecimentos \\
\cline { 2 - 2 } & Culpabilização \\
\cline { 2 - 2 } & Autoculpabilização \\
\hline
\end{tabular}

\section{Categoria Dimensão Emocional}

Esta categoria inclui duas subcategorias, nomeadamente a Auto-estigmatização e a Emoção expressa.

Diversos participantes referiram a Auto-estigmatização como uma barreira à resiliência familiar. Estes relataram que é comum a família da pessoa com esquizofrenia vivenciar sentimentos autoinfligidos de vergonha, perante 0 que pensam ser o que motiva os mitos culturais acerca das doenças mentais.

".... foi uma vergonha (...) ainda hoje quando ele sai à rua tenho certeza que todos olham para ele." E1

"Eu pensava que todos achavam que eu tinha feito algo de errado e que se iriam afastar de nós." E4

A emoção expressa também foi considerada pelos participantes como uma barreira para a resiliência familiar. A emoção expressa é uma medida que avalia 0 ambiente familiar através dos níveis expressos de crítica, hostilidade e sobre-envolvimento emocional.

"Deixamos de ter as quezílias todos os dias e passámos só a tê-las às semanas, já não há tantos gritos. " E7

"(...) não conseguem gerir isto e depois entram em conflito com ele. Ele ameaça eles e eles retribuem." E9

\section{Categoria Dimensão Relacional}

A categoria dimensão relacional é constituída pela subcategoria afastamento relacional. Os sintomas da doença conduzem à diminuição de competências sociais, que podem refletir-se na ausência e/ou disfuncionalidade de habilidades básicas de conversação e na manutenção das relações, o que exige por parte dos familiares 


\section{RESILIÊNCIA EM FAMÍLIAS DE PESSOAS COM ESQUIZOFRENIA: UM ESTUDO QUALITATIVO}

um maior esforço para manter a relação. Perante estas situações, muito familiares relataram um afastamento relacional, que em casos extremos levou ao termo da relação.

"O meu marido não teve capacidade para gerir isto e foi-se embora. Não aguentou ver o filho assim (...)"E3 "Já tentei que o pai falasse com ele mas nunca mais tiveram capazes de estar bem." E14

\section{Categoria Dimensão Racional}

A categoria dimensão racional é constituída por três subcategorias, nomeadamente 0 Défice de conhecimentos, a Culpabilização e a Auto-culpabilização.

Diversos participantes consideram que a realidade da esquizofrenia associada às consequências do lidar com a doença, produzem uma enorme sobrecarga e stress, tornando bastante difícil integrar a pessoa doente no seio da família. Para facilitar esta integração torna-se imperativo que os familiares adquiram conhecimentos sobre a doença.

"Se não perceberem a doença isto fica mais difícil. Depois de descobrir os vícios da doença a gente aprende a lidar com isto." E1

"(...) tento perceber os problemas da doença para não ser eu o problema." E6

Os participantes também identificaram a Culpabilização da pessoa doente como uma barreira para a resiliência familiar.

"Não sei o que ela andou por lá a fazer (...) nem sei o que acreditar. Possivelmente ela fez por lá algumas que levaram a que ficasse doente." E8

"Ele é que não soube escolher. Fez a vida que quis e depois ficou assim." E2

Contrastando com esta atitude, alguns dos participantes relataram a Auto-culpabilização como uma das principais barreiras à obtenção da resiliência familiar.

"A culpa disto tudo é minha, dei-lhe sempre tudo e agora ela não dá valor a nada. Nunca teve que se preocupar." E13

"Ainda cheguei a dizer à minha mulher que isto devia ter sido alguma coisa que fizemos." E12

\section{DISCUSSÃO}

A realização desta investigação possibilitou a identificação de três categorias principais que descrevem situações que surgiram frequentemente durante as entrevistas. As categorias, bem como as subcategorias resultantes da análise dos dados, permitem identificar e descrever as barreiras ao desenvolvimento da resiliência familiar.

As subcategorias que englobam maior número de unidades de registo foram o Défice de conhecimentos e a Emoção expressa. Este resultado pode ser indicador de que estas barreiras têm um impacto predominante no desenvolvimento da resiliência familiar.

A forma como os familiares interpretam a doença mental irá influenciar a sua prática de cuidados, a qual tem de ser encarada como um importante indicador de sucesso no processo de reabilitação e reinserção social da pessoa doente (Glynn, Cohen, Dixon, \& Niv, 2006; Sun et al., 2019).

0 desenvolvimento de uma doença crónica como a esquizofrenia é uma situação adversa tanto para a pessoa doente, como para a sua família. A gravidade das manifestações da doença, assim como a sua longa duração podem levar a que os familiares se tornem pessimistas em relação à evolução da doença, sendo bastante usual encontrar familiares cuidadores desmotivados e com sinais de sobrecarga perante 0 elevado volume de trabalho associado ao cuidar (Caqueo-Urízar et al., 2015; Fernandes, \& Vareta, 2019). Existem alterações na relação entre a pessoa doente e os seus familiares, que podem resultar em elevada emoção expressa, com conflitos interpessoais que conduzem à deterioração da relação (Lippi, 2016; Nuralita, Camellia, \& Loebis, 2019). À semelhança dos dados encontrados, outros autores também aferiram que os familiares cuidadores mais hostis ou hipercríticos recorrem preferencialmente a adjetivos negativos. Estes demonstram ser intolerantes perante os problemas da pessoa doente, recorrendo a estratégias impróprias e inflexíveis para lidar com as dificuldades, o que é pro- 
blemático, tendo em conta que as atitudes destes em relação à pessoa doente, são consideradas como um importante preditor de recidiva (Caqueo-Urízar et al., 2017; Nuralita et al., 2019).

Em consonância com as evidências demonstradas por outros investigadores, o défice de conhecimentos foi identificado pelos participantes como uma barreira à resiliência familiar. Sendo a resiliência um processo dinâmico e não uma característica específica de determinada pessoa, níveis mais elevados de conhecimentos sobre a doença irão permitir ao familiar cuidador demonstrar uma melhor capacidade de lidar com os processos de doença e ultrapassar essa adversidade (Fernandes, \& Castro, 2019; Grácio, Gonçalves-Pereira, \& Leff, 2016; Sin, \& Norman, 2013).

Outra das barreiras identificadas foi a Auto-estigmatização. 0 estigma afasta a família e a pessoa doente da sociedade, os familiares vêm a sua vida social limitada, não só pela absorção que a pessoa doente exige do cuidador, como pela rejeição, estabelecida pelo facto de serem encarados pela sociedade como agentes patogénicos da doença mental.

A identificação deste resultado está em concordância com os relatos de vários autores, que consideram que não se consegue eliminar a estigmatização desta doença na família, e este facto faz com que a família tenha sentimentos de vergonha (Corrigan, Larson, \& Rüsch, 2009; Koschorke et al., 2017).

A natureza e o estigma da doença mental podem provocar sentimentos de vergonha diante de outras pessoas. Para além de sentirem vergonha, os familiares de pessoas com doença mental, sentem culpa pela doença do seu familiar, o que leva ao desencadear do próprio estigma, sendo que a presença destes sentimentos acaba muitas vezes por levar os familiares a evitarem situações sociais (Rezayat, Mohammadi, Fallahi-Khoshknab, \& Sharifi, 2019; Sahar, \& Zaki, 2015). Outras investigações também identificaram que a falta de conhecimentos acerca da doença acabou por desencadear casos de auto-estigmatização (Caqueo-Urízar et al., 2017; Rezayat et al., 2019).

Considerando a barreira Afastamento relacional, esta foi identificada em diversas entrevistas. Para conviver em família, os seus elementos precisam de uma relação interpessoal, de compreensão em relação ao outro, pois são seres únicos, que diferem no modo de ser, agir e interagir. Com o diagnóstico da doença ocorre uma quebra nas expectativas dos familiares, que pode levar a alterações no relacionamento com a pessoa doente, conduzindo à deterioração da relação (Fernandes, \& Vareta, 2019; McCann et al., 2011). Associada a estas alterações, a perda ou não aquisição de competências sociais causadas pela doença, assim como a sobrecarga familiar originada pelo cuidar podem conduzir a um afastamento relacional, que em última instancia pode culminar com o abandono da pessoa doente (Chien, Leung, Yeung, \& Wong, 2013).

No que respeita às subcategorias Culpabilização e Autoculpabilização, estes resultados vão ao encontro com os dados encontrados por outros investigadores que aferiram que os indicadores de culpa são recorrentes nestas famílias, mesmo quando o diagnóstico psiquiátrico foi realizado há vários anos (Navarini, \& Hirdes, 2008; Silva, $\&$ Santos, 2009). Frequentemente os familiares de pessoas com esquizofrenia oscilam entre dois polos, a autoculpabilização pela doença, acreditando que o comportamento errático do doente é causado por algo que a família fez ou deixou de fazer, e a culpabilização da pessoa por ter adoecido, como se a doença tivesse despoletado em consequência de alguns comportamentos ou atitudes da pessoa doente (Cherry, Taylor, Brown, Rigby, \& Sellwood, 2017; Wasserman, de Mamani, \& Suro, 2012).

Existe evidência que os familiares das pessoas com esquizofrenia manifestam sentimentos de culpa, sendo a maior incidência na figura materna. Entre as principais causas culpabilização, identifica-se a atitude crítica que tinham em relação ao comportamento do doente, o desconhecimento da doença e a possível componente genética (Cherry et al., 2017; Wasserman et al., 2012).

\section{Limitações}

Esta investigação apresenta-se limitada em termos de transferibilidade, pois a técnica de amostragem representa maior facilidade operacional, mas resulta na incapacidade de fazer declarações gerais sobre a população, pelo que devem ser analisados novos pontos de vista em diferentes contextos. 


\section{RESILIÊNCIA EM FAMÍLIAS DE PESSOAS COM ESQUIZOFRENIA: UM ESTUDO QUALITATIVO}

Tal como em outros estudos qualitativos dependentes da colheita de dados através de entrevistas, existe a possibilidade da informação relatada ser diferentes da realidade. Isso pode ser devido à existência de vieses que influenciam as informações relatadas pelos participantes, como a falta de confiança em garantir o anonimato ou a proteção da identidade, dos seus valores ou crenças. No entanto, a confiabilidade na abordagem qualitativa é obtida pela riqueza de dados e, considerando que estes foram colhidos de vários participantes, considera-se pouco provável que tal tenha ocorrido.

\section{CONCLUSÃO}

Este estudo apresenta-se como uma das primeiras investigações que procuram identificar e explorar as barreiras à resiliência familiar. Partindo da narrativa dos participantes elaborou-se uma nova conceção, através da qual foram identificadas as barreiras à resiliência na perspectiva de 18 familiares cuidadores de pessoas com esquizofrenia.

De acordo com essa narrativa, identificaram-se três categorias de barreiras à resiliência familiar, nomeadamente Dimensão Emocional, Dimensão Relacional e Dimensão Racional que englobam seis subcategorias: Autoestigmatização, Emoção expressa, Afastamento relacional, Défice de conhecimentos, Culpabilização e Autoculpabilização.

A resiliência do familiar cuidador da pessoa com esquizofrenia parece ser passível de ser alcançado, para tal, é necessário bastante empenho por parte das equipas de saúde que os acompanham. 0 constructo de resiliência é um referencial importante para os profissionais de saúde, visando a possibilidade de voltar as suas práticas para 0 desenvolvimento da qualidade de vida e de comunidades resilientes, promovendo, assim, a saúde nos grupos e populações. A identificação de barreiras à resiliência familiar fornece uma visão das dificuldades vividas por esses cuidadores, permitindo que os profissionais de saúde se foquem nesses fatores e implementem estratégias que permitam remover ou minimizar a sua influência, potenciando as capacidades da pessoa para alcançar a adaptação positiva.

Considerando que esta é uma das primeiras abordagens ao fenómeno em causa, sugere-se a realização de mais pesquisas de modo a aumentar o conhecimento nesta área.

\section{REFERÊNCIAS BIBLIOGRÁFICAS}

Bardin, L. (2004). Análise de Conteúdo. Edições 70.

Caqueo-Urízar, A., Rus-Calafell, M., Craig, T. K., Irarrazaval, M., Urzúa, A., Boyer, L., ... Williams, D. R. (2017). Schizophrenia: Impact on Family Dynamics. Current psychiatry reports, 19(1), 2-8.

Caqueo-Urízar, A., Rus-Calafell, M., Urzúa, A., Escudero, J., \& Gutiérrez-Maldonado, J. (2015). The role of family therapy in the management of schizophrenia: challenges and solutions. Neuropsychiatric disease and treatment, 11, 145-151.

Cherry, M. G., Taylor, P. J., Brown, S. L., Rigby, J. W., \& Sellwood, W. (2017). Guilt, shame and expressed emotion in carers of people with long-term mental health difficulties: A systematic review. Psychiatry research, 249, 139-151.

Chien, W. T., Leung, S. F., Yeung, F. K., \& Wong, W. K. (2013). Current approaches to treatments for schizophrenia spectrum disorders, part II: psychosocial interventions and patient-focused perspectives in psychiatric care. Neuropsychiatric disease and treatment, 9, 1463-1481.

Corrigan, P. W., Larson, J. E., \& Rüsch, N. (2009). Self-stigma and the "why try" effect: impact on life goals and evidence-based practices. World psychiatry, 8(2), 75-81.

Davydov, D., Stewart, R., Ritchie, K., \& Chaudieu, I. (2010). Resilience and mental health. Clinical Psychology Review, 30(5), 479-495.

Eckermann, E. (2018). Resilience as a double-edged health promotion goal: exemples from Lao PDR. Elizabeth Eckermann 
School of Humanities and Social Sciences, Faculty of Arts and Education, Deakin University, Waurn Ponds, VIC 3217, Australia

Corresponding author. E-mail: liz.eckermann@deakin.edu.au

Search for other works by this author on:

Oxford Academic

PubMed

Google Scholar

Health Promotion International, 33(1), 123-131.

Fernandes, J. B., \& Castro, F. V. (2019). Variáveis sociodemográficas que se relacionam com a resiliência dos familiares das pessoas com esquizofrenia. International Journal of Developmental and Educational Psychology, 2(2), 45-52.

Fernandes, J. B., \& Vareta, D. A. (2019). Necessidades dos cuidadores informais da pessoa com demência em contexto de institucionalização de Iongo prazo. In R. Pocinho, \& N. P., Esperanza. Envelhecimento como perspetiva futura (611 - 623). Aranzadi Thomson Reuters.

Elizabeth Eckermann

Glynn, S. M., Cohen, A. N., Dixon, L. B., \& Niv, N. (2006). The potential impact of the recovery movement on family interventions for schizophrenia: opportunities and obstacles. Schizophrenia bulletin, 32(3), 451-463.

Grácio, J., Gonçalves-Pereira, M., \& Leff, J. (2016). What do we know about family interventions for psychosis at the process level? A systematic review. Fam Process, 55(1), 79-90.

Herrman, H., Stewart, D. E., Diaz-Granados, N., Berger, E. L., Jackson, B., \& Yuen, T. (2011). What is resilience?. Canadian journal of psychiatry, 56(5), 258-265.

Kim, G. M., Lim, J. Y., Kim, E. J., \& Kim, S. S. (2018). A model of adaptation for families of elderly patients with dementia: focusing on family resilience. Aging \& mental health, 22(10), 1295-1303.

Koschorke, M., Padmavati, R., Kumar, S., Cohen, A., Weiss, H. A., Chatterjee, S., ... Thornicroft, G. (2017). Experiences of stigma and discrimination faced by family caregivers of people with schizophrenia in India. Social science \& medicine, 178, 66-77.

Kuipers, E., Onwumere, J., \& Bebbington, P. (2010). Cognitive model of caregiving in psychosis. The British journal of psychiatry, 196(4), 259-265.

Li, Y., Qiao, Y., Luan, X., Li, S., \& Wang, K. (2019). Family resilience and psychological well-being among Chinese breast cancer survivors and their caregivers. European journal of cancer care, 28(2).

Lippi, G. (2016). Schizophrenia in a member of the family: Burden, expressed emotion and addressing the needs of the whole family. The South African journal of psychiatry, 22(1), 922.

Luthar, S. S., Cicchetti, D., \& Becker, B. (2000). The construct of resilience: A critical evaluation and guidelines for future work. Child Development, 71, 543-562.

McCann, T. V., Lubman, D. I., \& Clark, E. (2011). First-time primary caregivers' experience of caring for young adults with first-episode psychosis. Schizophrenia bulletin, 37(2), 381-388.

Melillo, A., \& Suárez-0jeda, E. (2005). Resiliência: descobrindo as próprias fortalezas. São Paulo, Brasil: Artmed, 2005.

Navarini, V., \& Hirdes, A. (2008). A família do portador de transtorno mental: identificando recursos adaptativos. Texto \& Contexto Enfermagem, 17(4), 680-688.

Nuralita, N., Camellia, V., \& Loebis, B. (2019). Relationship between Caregiver Burden and Expressed Emotion in Families of Schizophrenic Patients. Open access Macedonian journal of medical sciences, 7(16), 2583-2589.

O'Dwyer, S. T., Moyle, W., Taylor, T., Creese, J., \& Zimmer-Gembeck, M. (2017). In Their Own Words: How Family Carers of People with Dementia Understand Resilience. Behavioral sciences, 7(3), 57.

Patterson, J. L., \& Kelleher, P. (2005). Resilient school leaders: strategies for turning adversity into achievement. Association for Supervision and Curriculum Development. 


\section{RESILIÊNCIA EM FAMÍLIAS DE PESSOAS COM ESQUIZOFRENIA: UM ESTUDO QUALITATIVO}

Rezayat, F., Mohammadi, E., Fallahi-Khoshknab, M., \& Sharifi, V. (2019). Experience and the meaning of stigma in patients with schizophrenia spectrum disorders and their families: A qualitative study. Japan journal of nursing science, 16(1), 62-70.

Rutten, B., Hammels, C., Geschwind, N., Menne-Lothmann, C., Pishva, E., Schruers, K., ... Wichers, M. (2013). Resilience in mental health: linking psychological and neurobiological perspectives. Acta Psychiatrica Scandinavica, 128, 3-20.

Sahar, M., \& Zaki, R. (2015). Internalized Stigma of Mental IIIness among Schizophrenic Patients and Their Families (Comparative Study). Journal of Education and Practice, 6(12), 82-98.

Silva, G., \& Santos, M. A. (2009). Álbum de família e esquizofrenia: convivência em retrato. Psicologia em Estudo, 14(1), 83-91.

Sin, J., \& Norman, I. (2013). Psychoeducational interventions for family members of people with schizophrenia: a mixed-method systematic review. The Journal of clinical psychiatry, 74(12), e1145-e1162.

Sun, Y., Wang, M., Zhou, Y., Wang, L., Zhang, H., Lv, Y., ... Li, G. (2019). The mediating effect of family function and medication adherence between symptoms and mental disability among Chinese patients with schizophrenia: a cross-sectional study. Psychology, health \& medicine, 24(5), 559-569.

Teahan, Á., Lafferty, A., McAuliffe, E., Phelan, A., O'Sullivan, L., O'Shea, D., ... Fealy, G. (2018). Resilience in family caregiving for people with dementia: A systematic review. International journal of geriatric psychiatry, 33(12), 1582-1595.

Wasserman, S., de Mamani, A. W., \& Suro, G. (2012). Shame and guilt/self-blame as predictors of expressed emotion in family members of patients with schizophrenia. Psychiatry research, 196(1), 27-31. 